\title{
Textismos y ortografía. Percepción de los profesores en formación de la Generación Z
}

\author{
Coral I. Hunt-Gómez, Francisco Núñez-Román y Alejandro Gómez-Camacho \\ Universidad de Sevilla, Departamento de Didáctica de la Lengua y la Literatura y Filologías Integradas, Sevilla, España. \\ (correo-e: coralhuntg@us.es, fnroman@us.es, agomez21@us.es)
}

Recibido Sep. 17, 2019; Aceptado Nov. 10, 2019; Versión final Nov. 28, 2019, Publicado Abr. 2020

\begin{abstract}
Resumen
Bajo la hipótesis de que los hablantes jóvenes son más tolerantes hacia los textismos y su inclusión en el aula, se analiza la percepción de la influencia de los textismos en la ortografía de una muestra de 264 profesores en formación pertenecientes a la Generación Z, alfabetizados en un entorno de escritura digital. Los resultados indican que, aunque los participantes consideran que los textismos se deben incluir en el aula como recurso didáctico, simultáneamente piensan que son perjudiciales para la ortografía y los deben corregir. Los resultados del estudio muestran también que la percepción de los participantes varía según las diferentes categorías de textismos. Los valores normativos de los grafemas en la escritura culta se consideran más dañinos para la ortografía. Sin embargo, los elementos multimodales y los que no alteran la relación entre fonemas y grafemas gozan de una mayor tolerancia. Se concluye que desde la universidad se debe dotar a los profesores en formación de las competencias necesarias para que sean capaces de superar los prejuicios asociados a los textismos e incorporarlos a su práctica docente.
\end{abstract}

Palabras clave: textismos; ortografía; generación Z, escritura académica; español; formación universitaria.

\section{Textisms and spelling. Perception of pedagogy students of the Z Generation}

\begin{abstract}
Following the hypothesis that younger university students adopt a more tolerant attitude towards textisms and their introduction in the classroom, this work analyses the perception of textisms on spelling of 264 students op pedagogy belonging to the $Z$ Generation, educated in a digital writing environment. Results show that even if textisms are considered a didactic resource to be introduced in the classroom, simultaneously, they were are believed prejudicial for spelling, and consequently, participants believed that they must be corrected. Results also indicate that participants' attitude varies depending on the different categories of textisms: those textisms differing from the normative values of graphemes in the academic writing are thought to be more harmful to spelling. However, multimodal elements and those not modifying the relationship between phonemes and graphemes enjoy a higher degree of tolerance. One of the main conclusions of the study is that the university should provide future teachers with the necessary competences to overcome preconceived ideas and include new writing forms in the classroom.
\end{abstract}

Keywords: textisms; spelling; generation Z; literacy; Spanish; higher education. 


\section{INTRODUCCIÓN}

En las últimas décadas, la sociedad ha experimentado dos grandes revoluciones. La primera, a finales del siglo XX, fue la globalización que vino de la mano de la democratización de Internet y su incorporación a la a la vida diaria de millones de personas. La segunda revolución fue la de los teléfonos inteligentes que, acompañados de otros dispositivos móviles con funciones parecidas, permiten un acceso inmediato a creciente número de posibilidades con artefactos que se llevan cómodamente encima (Pedrero-Pérez et ál., 2018). Estas herramientas permiten nuevas vías de relación entre las personas y son relevantes en multitud de ámbitos (Garrote-Rojas, et al., 2018).En consecuencia, se ha incorporado a la educación superior una nueva generación de estudiantes nacidos entre 1995 y 2012 que se ha alfabetizado y ha cursado la educación primaria y secundaria en un contexto digital, donde la comunicación se establece por medios que hasta ahora no habían existido, la llamada Generación Z (Prensky,2009).

Aunque existen multitud de términos con los que designar a aquellos nacidos en la era digital y, a pesar de que, conceptualmente, no se cuente con una definición exhaustiva ni con unos confines temporales claros que la enmarquen (Jones, et al., 2010), para el propósito que persigue este trabajo se considera que todos los nacidos a partir de 1995 pertenecer a la Generación Z. Este estudio se centra en la percepción de futuros profesores de español pertenecientes a la Generación Z, que han de afrontar la adquisición de la norma culta de escritura en convivencia con una nueva norma de escritura digital (Álvarez y Taboada, 2016). Esta norma digital es la norma escrita que se suele usar en la comunicación instantánea y que cuenta con algunas características propias que se describen en los siguientes párrafos. Estudios recientes muestran que los profesores en formación mantienen una actitud abierta hacia la incorporación de las redes sociales y la comunicación digital para la enseñanza de lenguas, entre ellas el español (Carpenter et al., 2016).

La mensajería instantánea ya es parte fundamental de nuestras vidas y cada vez es más fácil acceder a ella desde distintas localizaciones. Un reciente informe (Fundación Telefónica, 2019) señala que se ha producido un notable incremento en el uso de teléfonos inteligentes y que el $95,1 \%$ de la población prefiere este medio, llegando incluso a superar la comunicación en persona. La tendencia se acentúa en el caso de los jóvenes, cuyos hábitos se caracterizan por su preferencia por la mensajería instantánea sobre otras formas de comunicación y su capacidad para trabajar y colaborar de manera virtual. Según este mismo informe, en 2018, un 96,8\% de los jóvenes españoles entre 14 y 24 años utilizó diariamente WhatsApp como canal de comunicación predilecto.

Este auge del uso de la mensajería instantánea ha propiciado la aparición de un nuevo código escrito, es decir, una nueva norma digital que se denomina digitalk (Turner, 2010) textise (Johnson, 2015), y que se caracteriza porque difiere de la norma estándar de escritura, también en castellano (Gómez-Camacho, et al., 2018). La ortografía y el uso especial que se hace de los caracteres en este nuevo código ha dado lugar a los denominados textismos, en lengua inglesa textisms. De Jonge y Kemp (2012) y Plester et al. (2009) destacan, entre otros ejemplos, como textismos más relevantes en lengua inglesa la falta de uso de mayúsculas o signos de puntuación, el uso de números o letras homófonos (Vázquez-Cano, et al., 2015), abreviaturas, onomatopeyas, emoticonos o símbolos tipográficos. Por su parte, Bernicot et ál. (2014) establecen una distinción muy interesante entre los textismos que son consistentes en la relación entre fonemas y grafemas, frente a los que alteran esta relación fijada por la norma culta de escritura en lengua francesa. De acuerdo con Mas y Zas (2012), los textismos presentan dos fenómenos básicos: la abreviación y la homofonía. En estudios más recientes se incluye una categorización, que además de haber sido diseñada específicamente para el estudio de los textismos en lengua española, es mucho más exhaustiva (Gómez-Camacho, et al., 2018). Esta categorización (Tabla 1) se divide en las siguientes categorías: repeticiones, omisiones, grafemas no normativos, textismos léxicos y elementos multimodales.

El interés por la norma digital de escritura trasciende el ámbito lingüístico y plantea una dimensión pedagógica que afecta de lleno a la educación superior, singularmente a la formación universitaria en Ciencias de la Educación. Thurlow (2006) señalaba algunas ideas preconcebidas en cuanto a la comunicación digital e instaba a desarrollar estudios centrados en los aspectos lingüísticos y ortográficos. Desde la comunidad científica se ha analizado la relación entre los textismos y la escritura académica culta, que alcanza su máxima expresión en la competencia ortográfica (De Jonge y Kemp, 2012; Drouin y Driver, 2014; Kemp y Abbie, 2017; Gómez-Camacho et al., 2018). Varias investigaciones recogen que, de manera general, los textismos son considerados como una amenaza para la norma tradicional y más aún, en el caso de que los utilicen personas que están en proceso de adquisición de la escritura (De Jonge y Kemp, 2012; Thurlow y Poff, 2013). En algunos casos, incluso se enumeran consecuencias del uso de las nuevas formas de escritura entre estudiantes en proceso de formación como la reducción de las frases y reglas ortográficas omitiendo reglas lingüísticas, la influencia sobre el desarrollo del lenguaje y la escritura, y la poca capacidad de escritura formal (Suárez-Loor, 2015). 
Tabla 1. Categorización de textismos (Gómez-Camacho, Hunt-Gómez, Valverde-Macías, 2018)

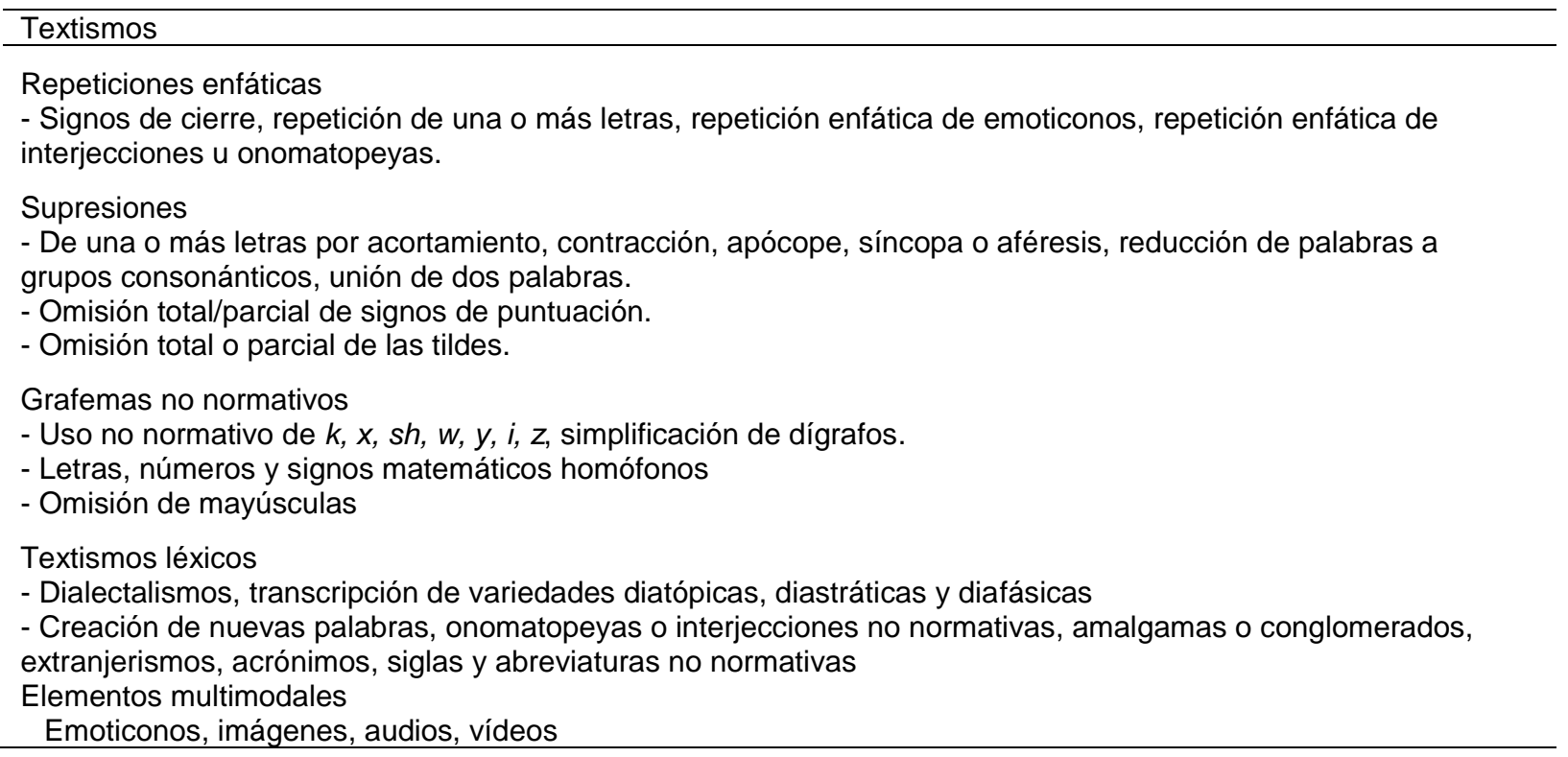

Numerosos estudios sobre la influencia de los textismos en la ortografía y en la adquisición de la escritura han demostrado que el uso de la mensajería instantánea no tiene repercusiones negativas sobre la ortografía, entre ellos los realizados por Bouillaud et al., (2007) y Lanchantin et al., (2014). En el caso concreto de la lengua española, nuevas investigaciones concluyen que tampoco se da una relación negativa directa entre el uso de textismos y la competencia ortográfica de hablantes que cursan la educación universitaria (Gómez-Camacho et al., 2018). Pascual (2013) va más allá y afirma que las personas que tienen buenas destrezas escritoras poseen la flexibilidad necesaria para adaptarse y utilizar los nuevos códigos de escritura digital. Paradójicamente, se ha generalizado la percepción de que el uso de una norma de escritura digital alternativa a la norma académica culta es un obstáculo para la adquisición de la competencia ortográfica, especialmente del alumnado adolescente (Pinedo, 2016; Cadena Ser, 2017).

La investigación sobre este tema en el contexto universitario ha recogido numerosas intervenciones que fomentan el uso de las nuevas formas de escritura digital como una oportunidad en la formación de docentes (Risser, 2013; Carpenter y Krutka, 2015; Carpenter y Green, 2017, Monroy, et al., 2018). En el ámbito de la Didáctica de lenguas resulta imprescindible integrar las nuevas tendencias y formas de comunicación multimodales en el aula, más aún cuando constituye, con un amplio margen, el medio de comunicación escrita más frecuente. En este contexto, la formación en Ciencias de la Educación debe ofrecer herramientas para reaccionar ante los retos que presentan las nuevas formas de escritura (Carpenter y Green, 2017).

En un estudio previo (Gómez-Camacho et al., 2018), llegamos a la conclusión de que los hablantes más jóvenes se mostraban más tolerantes con el uso de una norma digital alternativa a la norma académica culta en la comunicación escrita a través de teléfonos inteligentes. Este trabajo desarrolla esa línea de investigación, centrada ahora en los profesores de español de educación primaria en formación pertenecientes a la denominada Generación Z Se pretende identificar su percepción sobre la dimensión educativa del uso de textismos en la comunicación a través de teléfonos inteligentes, y su relación con la adquisición de la competencia lingüística y ortográfica en el alumnado adolescente. Para ello se han seleccionado estudiantes de Ciencias de la Educación de la universidad de Sevilla, una institución con más de quinientos años de antigüedad, nacidos a partir de 1995, que habían realizado prácticas en las aulas de Educación Primaria en el último año. Se trata, en consecuencia, de futuros profesionales que habrán de afrontar la alfabetización y la educación lingüística de un alumnado nativo digital, en unas condiciones similares a las que ellos experimentaron cuando cursaron la educación obligatoria. No hay por tanto un salto generacional comparable al que enfrenta el profesorado en formación de mayor edad, y se espera que la formación universitaria en Ciencias de la Educación les haya dotado de herramientas para enfrentarse a un reto profesional aún por resolver (Álvarez y Taboada, 2016).

\section{METODOLOGÍA}

Se trata de un estudio sincrónico no experimental basado en una encuesta que arroja resultados descriptivos. La descripción de la metodología se expone en cuatro subsecciones: preguntas de investigación, participantes, instrumento, y procedimiento 


\section{Preguntas de investigación}

A partir de las investigaciones previas, pretendemos determinar si los profesores en formación que han sido nativos digitales aceptan la inclusión de los textismos como un elemento coadyuvante para la alfabetización y la adquisición de la competencia ortográfica, en el contexto del aula de lengua española en la educación obligatoria. Surgen en consecuencia dos interrogantes fundamentales a los que este trabajo pretende dar respuesta: 1 . ¿Consideran los participantes en el estudio que la norma digital es una oportunidad para la alfabetización del alumnado, o por el contrario supone una amenaza para la adquisición de la norma culta de escritura en español?; y 2. ¿Se establecen diferencias en la percepción de los profesores en formación entre los diferentes tipos de textismos recogidos en la clasificación de la Tabla 1 ?

\section{Participantes}

Para el estudio de campo se recurrió a estudiantes de último curso de Ciencias de la Educación de la Universidad de Sevilla que realizaban prácticas profesionales en el aula de lengua española de Educación Primaria. La muestra estuvo conformada por total de 264 sujetos $(n=264)$, lo que supone un $56,5 \%$ del total de población matriculada en el último curso, que ascendía a 480 alumnos. Todos los participantes nacieron a partir de 1995 por lo que contaban con edades comprendidas entre 20 y 22 años. En cuanto al sexo, la muestra está compuesta por un $75,7 \%$ de mujeres y un $24,3 \%$ de hombres.

\section{Instrumento}

El instrumento utilizado en esta investigación se basa en la escala titulada "Textismos y norma escrita en la enseñanza del español" sobre textismos y norma digital que diseñamos para una investigación anterior (Gómez-Camacho et al., 2018). Una adaptación de esta escala también fue utilizada en investigaciones paralelas para la lengua italiana y la lengua portuguesa (Gómez-Camacho et al., 2017). De los 39 ítems originales se seleccionaron 16 relacionados con las preguntas de investigación de este trabajo, en una escala tipo Likert que utiliza un formato de respuesta de cinco alternativas con un rango de 1 («Totalmente en desacuerdo») a 5 («Totalmente de acuerdo»). La escala se recoge en la Tabla 2. Se solicitó también a los participantes que indicaran su edad y su sexo.

Tabla 2. Escala utilizada (Adaptación de Gómez-Camacho, Hunt-Gómez, Valverde-Macías, 2018)

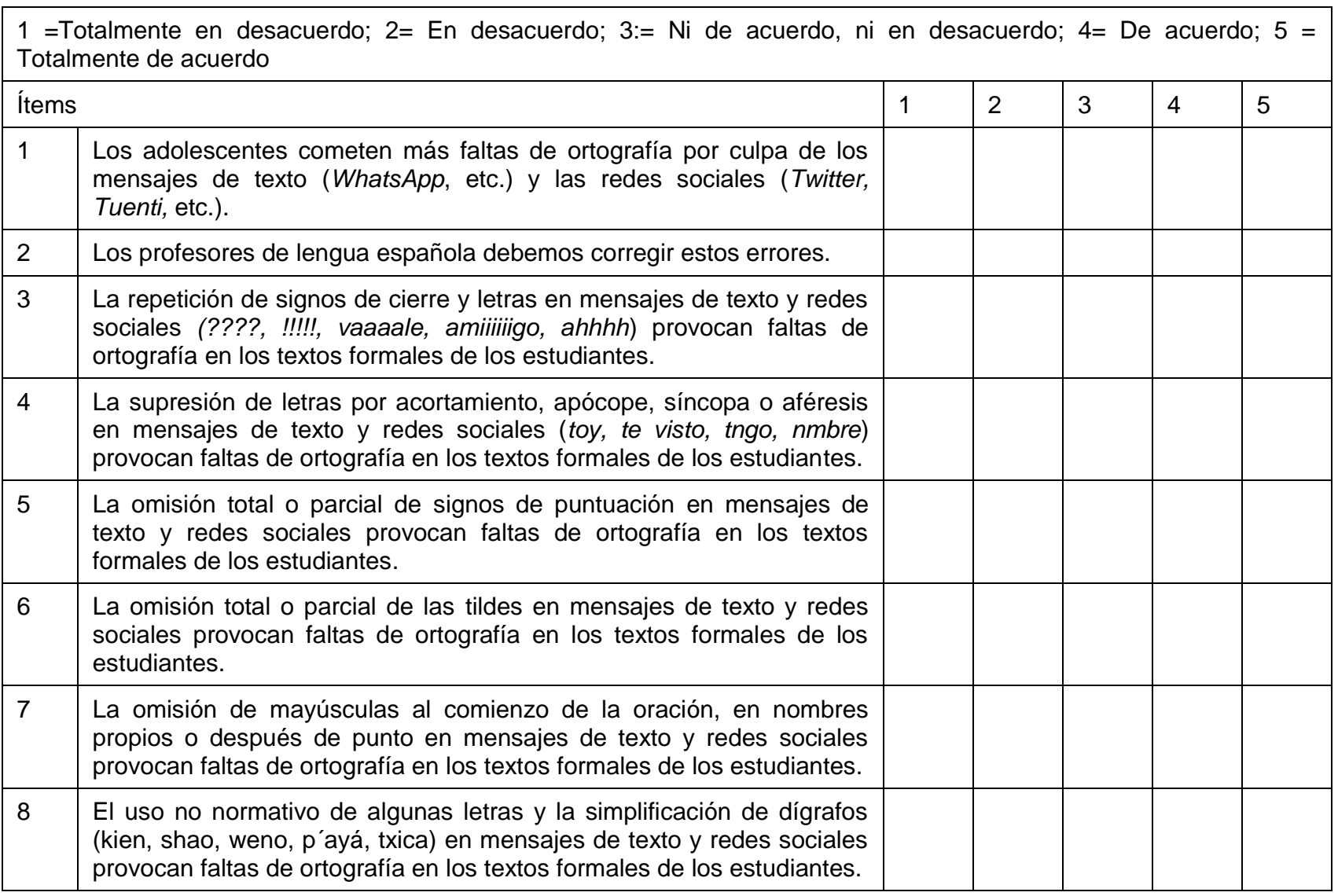


Tabla 2: Continuación.

\begin{tabular}{|c|c|c|c|c|c|c|}
\hline \multicolumn{2}{|c|}{ Ítems } & \multirow[t]{2}{*}{1} & \multirow[t]{2}{*}{2} & \multirow[t]{2}{*}{3} & \multirow[t]{2}{*}{4} & \multirow[t]{2}{*}{5} \\
\hline 9 & $\begin{array}{l}\text { El uso de letras y signos matemáticos por su nombre ( } x \text { favor, + cosas, } \\
d a 2, t \text { veo, } d \text { nada) en mensajes de texto y redes sociales provocan } \\
\text { faltas de ortografía en los textos formales de los estudiantes. }\end{array}$ & & & & & \\
\hline 10 & $\begin{array}{l}\text { La omisión intencionada de h y los dialectalismos intencionados (asta, } \\
\text { e pelao, ceñío, improvisao, toas, to rayá, pegá, ziii, dise) en mensajes } \\
\text { de texto y redes sociales provocan faltas de ortografía en los textos } \\
\text { formales de los estudiantes. }\end{array}$ & & & & & \\
\hline 11 & $\begin{array}{l}\text { La creación de nuevas palabras, onomatopeyas e interjecciones no } \\
\text { normativas (pfff, pirfii, sip, jartao, wasap, potito, lok) en mensajes de } \\
\text { texto y redes sociales provocan faltas de ortografía en los textos } \\
\text { formales de los estudiantes. }\end{array}$ & & & & & \\
\hline 12 & $\begin{array}{l}\text { El uso de emoticonos y elementos multimedia (imágenes, audios y } \\
\text { vídeos) en mensajes de texto y redes sociales provocan faltas de } \\
\text { ortografía en los textos formales de los estudiantes. }\end{array}$ & & & & & \\
\hline 13 & $\begin{array}{l}\text { La incorporación de textismos como recurso didáctico en el aula de } \\
\text { lengua española de educación secundaria es necesaria en el contexto } \\
\text { actual. }\end{array}$ & & & & & \\
\hline 14 & $\begin{array}{l}\text { Estos textismos son discrepancias intencionadas de la norma de } \\
\text { escritura culta y son aceptables si se limitan a la comunicación no } \\
\text { formal en mensajes de texto y redes sociales. }\end{array}$ & & & & & \\
\hline 15 & $\begin{array}{l}\text { Los nuevos géneros de escritura en la comunicación por Internet } \\
\text { cambiarán la norma académica culta de escritura en un futuro próximo. }\end{array}$ & & & & & \\
\hline 16 & Estos cambios supondrían un empobrecimiento de nuestra lengua. & & & & & \\
\hline
\end{tabular}

La validez del instrumento original se determinó tanto en el plano teórico como en el plano empírico. En lo relativo a la validez teórica, una versión inicial de dicha escala fue presentada a un grupo de cinco profesores e investigadores de didáctica de la lengua de la Universidad de Sevilla; en cuanto a la validez empírica, se determinó considerando la validez de constructo de la escala para lo cual hemos utilizado el escalamiento multidimensional PROXSCAL (Borg et al., 2013). Se realizó un estudio cuantitativo, a partir de un muestreo exploratorio, no probabilístico por conveniencia. Además, se calculó el coeficiente de alfa de Cronbach del cuestionario final formado por 16 preguntas cerradas con un resultado de ,860, que muestra la elevada consistencia interna de la escala utilizada en este estudio.

\section{Procedimiento}

La recogida de datos se produjo en el curso académico 2016/2017; la escala se suministró individualmente a los participantes en el estudio a través de Google Forms, no existiendo límite de tiempo para su cumplimentación. El programa de realización de encuestas generó los datos descriptivos que sirvieron para analizar de manera inicial la percepción de los distintos textismos de los sujetos. Posteriormente, los datos se exportaron y fueron analizados mediante el uso del programa informático estadístico SPSS.

\section{RESULTADOS}

Las preguntas de investigación planteadas obtuvieron los resultados que se presentan en lo que sigue. Para mayor claridad se repite la pregunta y se analizan los resultados obtenidos.

Pregunta de investigación 1. ¿Consideran los participantes en el estudio que la norma digital es una oportunidad para la alfabetización del alumnado, o por el contrario supone una amenaza para la adquisición de la norma culta de escritura en español?

Los participantes consideran que los textismos suponen una amenaza para la norma culta escrita en español y deben ser corregidos en el aula de lengua. Así, en el ítem 1, los participantes del estudio están de acuerdo en que la norma digital provoca faltas $(\dot{x}=4,10)$ y que, en consecuencia, los profesores de español deben corregirla $(\dot{x}=4,45)$. En términos de aceptación o rechazo de los textismos, si consideramos que las opciones 1 y 2 en la escala propuesta suponen desacuerdo, mientras que 4 y 5 implican acuerdo, el $73.9 \%$ 
de los participantes relacionan textismos y faltas (frente al $8,2 \%$ que los diferencia) y el $84,3 \%$ considera que es su labor como profesor de lengua española corregirlos. Sin embargo, cuando se formula la misma pregunta desde un punto de vista normativo, independiente por tanto del contexto educativo (ítem 14), los participantes en el estudio aceptan sin problemas que los textismos son discrepancias intencionadas de la norma de escritura culta y son aceptables en mensajes de texto $(\dot{x}=3,50)$ y solo un $9,7 \%$ de los participantes rechaza los textismos. De forma paralela, se acepta el uso de textismos como recurso didáctico $(\dot{x}=3,21)$ y solo es rechazado por $22.7 \%$ de los participantes.

Esta contradicción en la percepción de los participantes sobre dos caras del mismo fenómeno se confirma al establecer la correlación de Pearson entre las variables 1, 2, 13 y 14 del estudio que ofrecen resultados no significativos que oscilaron entre,- 101 y ,085 $\left(n=264,{ }^{*} p<.05,{ }^{* *} p<.01\right)$. Con respecto a los ítems 15 y 16 sobre la influencia de los textismos en la norma culta y si esta influencia será perjudicial para la misma, también resulta contradictorio que solo el $36,9 \%$ considere que esa relación se producirá, mientras que el $60,5 \%$ afirma que será negativa. De nuevo, las correlaciones con los valores de los ítems analizados en este apartado no resultan relevantes, excepto en la correlación de Pearson poco significativa entre el ítem 16 con el ítem $1\left(, 219^{* *}, \mathrm{n}=264,{ }^{*} \mathrm{p}<.05,{ }^{* *} \mathrm{p}<.01\right)$ y con el ítem 13 de la escala $\left(-, 223^{* *}, \mathrm{n}=264,{ }^{*} \mathrm{p}<.05\right.$, $\left.{ }^{* *} p<.01\right)$.

Pregunta de investigación 2. ¿Se establecen diferencias en la percepción de los profesores en formación entre los diferentes tipos de textismos recogidos en la clasificación de la Tabla 1?

Los participantes en el estudio establecen diferencias significativas en su percepción de los textismos en relación con las faltas de ortografía cometidas por el alumnado en sus textos académicos en lengua española. La puntuación media en la escala referida a los ítems $4,5,6,7,8$ y 10 oscila entre $\dot{x}=4,09$ y $\dot{x}=$ 4,29 , con acuerdo entre el $82,5 \%$ y el $74,3 \%$ en que estos textismos provocan faltas de ortografía. Frente a ellos, los ítems 3, 9 y 11 no alcanzan el 4 de media ni el acuerdo de al menos el $70 \%$ de los participantes, lo que supone una aceptación significativamente menor. Por el contrario, el ítem 12 referido al uso de elementos multimodales no se relaciona con las faltas de ortografía $(\dot{x}=2,04)$, lo que es corroborado por un $66,8 \%$ de los participantes en el estudio.

A nivel correlacional, el coeficiente de correlación de Pearson, que aborda las posibles relaciones entre las variables que responden a la segunda pregunta de investigación, confirma la coherencia de los resultados y la diferente percepción de los participantes entre los textismos que se corresponden con las grafías cultas del español (ítems 4, 5, 6, 7, 8 y 10), los textismos que se relacionan con signos auxiliares o unidades del plano léxico (ítems 3,9 y 11), y las unidades multimodales que no emplean grafemas ni signos auxiliares de la norma culta de escritura (ítem 12). Se establece en consecuencia una diferencia entre los textismos que alteran la relación entre fonemas y grafemas de la norma culta de escritura académica, y los que suponen una nueva forma de escribir con elementos que no se usan en los textos académicos.

Tabla 3. Correlación de Pearson entre las variables 3-12 $\left(n=264,{ }^{*} p<.05,{ }^{* *} p<.01\right)$.

\begin{tabular}{|c|c|c|c|c|c|c|c|c|c|}
\hline & 3 & 4 & 5 & 6 & 7 & 8 & 9 & 10 & 11 \\
\hline 4 &, $650^{* *}$ & & & & & & & & \\
\hline 5 &, $617^{\star \star}$ &, $705^{\star *}$ & & & & & & & \\
\hline 6 &, $559^{\star *}$ &, $661^{* *}$ &, $704^{* *}$ & & & & & & \\
\hline 7 &, $577^{\star *}$ &, $645^{\star *}$ &, $658^{* *}$ &, $705^{\star *}$ & & & & & \\
\hline 8 &, $582^{\star *}$ &, $689^{* *}$ &, $681^{* *}$ &, $740^{\star *}$ &, $756^{\star *}$ & & & & \\
\hline 9 &, $529^{\star *}$ &, $672^{* *}$ &, $620^{* *}$ &, $623^{* *}$ &, $637^{* *}$ &, $709^{* *}$ & & & \\
\hline 10 &, $520^{\star *}$ &, $661^{* *}$ &, $655^{\star *}$ &, $695^{\star *}$ &, $632^{* *}$ &, $765^{\star *}$ &, $679^{* *}$ & & \\
\hline 11 &, $610^{\star *}$ &, $634^{* *}$ &, $618^{\star *}$ &, $543^{* *}$ &, $648^{\star *}$ &, $620^{* \star}$ &, $751^{\text {** }}$ &, $580^{\star \star}$ & \\
\hline 12 &, $232^{* *}$ &, $259^{\star *}$ &, $235^{\star \star}$ &, $151^{*}$ &, $274^{* *}$ &, $192^{* *}$ &, $302^{* *}$ & ,120 & \\
\hline
\end{tabular}

\section{DISCUSIÓN}

Con respecto a la consideración de la norma digital como una oportunidad o una amenaza para la norma de escritura culta, los resultados muestran que los futuros profesores de la Generación Z no tienen una visión coherente en cuanto a la relación de los textismos con las faltas de ortografía. La gran mayoría de los participantes considera que los textismos son discrepancias intencionadas de la norma culta siempre que se 
limiten a la comunicación no formal en mensajes de texto y redes sociales y que deben incluirse en el aula de lengua como recurso didáctico. Paradójicamente, la opinión de los participantes resulta categórica en cuanto a la relación negativa entre las faltas de ortografía y los textismos y, en consecuencia, señalan que los profesores deben corregirlos.

En relación con el aprendizaje de la norma culta de escritura en español, la principal conclusión a la que se llega es que los futuros profesores de la Generación Z consideran que las nuevas formas de escritura digital son una oportunidad para la enseñanza de la lengua y que deben ser incluidas como recursos educativos en el aula, lo que entra en contradicción con su percepción negativa sobre los textismos en general y su influencia en la ortografía. No obstante, aunque la percepción de los textismos en bloque sea negativa, al analizar los distintos tipos de textismos de manera aislada se aprecia que, los futuros maestros pertenecientes a la Generación $Z$ no perciben todos de textismos de la misma forma. Este resultado es coherente con nuestros estudios previos sobre las percepciones, tanto las de adolescentes (Vázquez-Cano et al., (2015) como las de los futuros profesores tanto nativos como inmigrantes digitales (Gómez-Camacho et al., 2018).

Los datos muestran que los textismos que utilizan grafemas no normativos, que omiten mayúsculas o tildes se identifican con faltas de ortografía que generan problemas de aprendizaje. Este resultado es consistente con las investigaciones realizadas para la lengua francesa en la que se concluye que los textismos que no siguen la relación tradicional entre fonemas y grafemas son los que se consideran más perjudiciales para la ortografía (Bernicot et ál., 2014). Por el contrario, los textismos mejor valorados son aquellos que no entran en conflicto con la norma de escritura culta y que, por tanto, no se asocian a faltas de ortografía. En este caso pueden considerarse como una seña de identidad, en línea con lo propuesto por Mansera (2016). Resulta destacable el caso de los elementos multimodales, considerados como nuevas unidades lingüísticas no verbales que no se utilizan en la escritura tradicional; en consecuencia, los maestros en formación de la Generación Z apenas consideran que tengan efectos negativos para la ortografía.

Los resultados de este estudio no permiten concluir que futuros maestros de la Generación Z muestren una percepción más favorable hacia la inclusión de los textismos en el aula de lengua española, ni que acepten nuevas normas de escritura discrepante con la norma académica culta. Esto puede deberse a que, a pesar de que los participantes a través de su uso cotidiano perciban la necesidad y la utilidad de los nuevos códigos, desde el ámbito de la formación universitaria no se les hayan proporcionado los recursos didácticos necesarios para incorporar estas nuevas formas de escritura de manera eficaz a su futura práctica docente. Por tanto, al carecer de estrategias pedagógicas adecuadas, se ven arrastrados por los perjuicios generalizados en cuanto a las nuevas formas de escritura y su relación con la ortografía. Desde la formación universitaria, especialmente la de los futuros maestros, se deben ofrecer las herramientas y las estrategias para superar las concepciones arbitrarias y proporcionar a los futuros egresados directrices para adaptar su docencia a los cambios de la sociedad. Algunas propuestas van desde la coordinación entre las enseñanzas que componen las materias del currículo de formación de profesorado dedicadas a la inserción de las TIC en el aula con las de Didáctica de la Lengua o el siempre necesario acercamiento de la realidad lingüística al aula mediante el uso o el estudio de los mensajes digitales y sus distintos aspectos multimodales.

Además de examinar el resultados de la coordinación de materias que integren las TIC, la Lengua y la Didáctica, en el futuro se espera poder realizar un estudio con una muestra mayor que permita dar solidez a los resultados obtenidos puesto que el estudio de los textos digitales y las nuevas formas de comunicación constituyen uno de los grandes retos a los que se enfrenta la Didáctica de la Lengua en la actualidad.

\section{CONCLUSIONES}

A partir del análisis de los resultados obtenidos y de la discusión planteada se puede concluir lo siguiente: 1) los profesores en formación pertenecientes a la Generación Z no muestran una opinión coherente sobre la influencia de los textismos en la ortografía, puesto que aunque consideran que deben incluirse en el aula como recurso didáctico, los perciben como perjudiciales para la ortografía y piensan que deben corregirlos; 2) los profesores en formación pertenecientes a la Generación $Z$ entienden que no todos los textismos tienen la misma influencia sobre la ortografía, así, no consideran que los elementos multimodales le afecten en absoluto; 3) la formación universitaria dirigida a futuros profesores debe proporcionarles recursos, herramientas y estrategias para que adapten su docencia a la comunicación escrita de la sociedad actual y superen las ideas preconcebidas.

\section{REFERENCIAS}

Álvarez, G. y Taboada, M. B., Propuestas Didácticas Mediadas por Tecnologías Digitales para el Desarrollo de Competencias de Lectura y Escritura Académicas. https://doi.org/10.21500/22563202.2336, Rev. Guillermo de Ockham, 14(2), 83-91 (2016) 
Bernicot, J., Goumi, A., Bert-Erboul, A., Volckaert-Legrier, O., How Do Skilled and Less-Skilled Spellers Write Text Messages? A longitudinal study. https://doi.org/10.1111/jcal.12064. Journal of Computer Assisted Learning 30, 559-576 (2014)

Borg, I., Groenen, P. y Mair, P., Applied Multidimensional Scaling. Springer: Heidelberg (2013)

Bouillaud, C., Chanquoy, L. y Gombert, J., Cyberlangage et Orthographe : Quelles Effets sur le Niveau Ortographique des Élèves de CM2, 5e et 3e?, https://doi.org/10.3917/bupsy.492.0553. Bulletin de Psychologie, 492, 553-365 (2007)

Cadena Ser., Muchos Errores Ortográficos de los Niños Llegan por el Uso de Internet y las Redes Sociales. (2017)

Carpenter, J.P. y Green T.D., Mobile Instant Messaging for Professional Learning: Educators' perspectives on and uses of Voxer. https://doi.org/10.1016/j.tate.2017.08.008. Teaching and Teacher Education, 68, 53-67 (2017)

Carpenter, J. P. y Krutka, D. G., Engagement through Microblogging: Educator Professional Development via Twitter. https://doi.org/10.1080/19415257.2014.939294, Professional Development in Education, 41(4), 707-728 (2015)

Carpenter, J.P., Tur, G. y Marín, V.I. What do U.S. and Spanish Pre-Service Teachers Think about Educational and Professional Use of Twitter? A Comparative Study. https://doi.org/10.1016.j.tate.2016.08.011 Teaching and Teacher Education, 60, 131-146 (2016)

De Jonge, S. y Kemp, N., Text-Message Abbreviations and Language Skills in High School and University Students. https://doi.org/10.1111/j.1467-9817.2010.01466.x, Journal of Research in Reading, 35 (1), 49-68 (2012)

Drouin, M. y Driver, B. Texting, Textese and Literacy Abilities: A Naturalistic Study. https://doi.org/10.1111/j.14679817.2012.01532.x, Journal of Research in Reading, 37(3), 250-267 (2014)

Fernández-Cruz, F.J., y Fernández-Díaz, M.J., Los Docentes de la Generación Z y sus Competencias Digitales. https://doi.org/10.3916/C46-2016-10. Comunicar. 46, XXIV, 97-105 (2016)

Fundación Telefónica. La Sociedad de la Información en España. (2019)

Garrote-Rojas, D., Jiménez-Fernández, S., y Gómez-Barreto, M.I., Problemas Derivados del Uso de Internet y el Teléfono Móvil en Estudiantes Universitarios. https://doi.org/10.4067/S0718-50062018000200099. Formación Universitaria, 11(2), 99-108. (2018)

Gómez-Camacho, A., Hunt-Gómez, C.I., Valverde-Macías, A., Textism, Texting, and Spelling in Spanish. https://doi.org/10.1016/j.lingua.2017.09.004. Lingua, 201, 92-101 (2018)

Gómez-Camacho, A., Núñez-Román, F., y Perera-Rodríguez, V.H., Testismi, Messaggistica e Didattica dell'Italiano: Percezioni degli Studenti Universitari, https://doi.org/10.3280/Cad2016-002006. Cadmo: giornale italiano di pedagogia sperimentale, 2. 57-74 (2017)

Jones, C.; Ramanau, R.; Cross, S. Y y Healing, G., Net generation or Digital Natives: Is there a distinct new generation entering university? https://doi.org/10.1016/j.compedu.2009.09.022. Computers \& Education, 54(3). 722-732 (2010).

Johnson, G. M., The Invention of Reading and the Evolution of Text. Journal of Literacy and Technology, 16 (1), 107-128 (2015)

Kemp, N. y Abbie, G., Txting Across Time: Undergraduates' Use of 'Textese' in Seven Consecutive First-Year Psychology Cohorts, https://doi.org/10.1080/17586801.2017.1285220, Writing Systems Research,1-16 (2017)

Lanchantin, T., Simoës-Perlant, A. y Largy, P., Good Spellers Write More Textism than Bad Spellers in Instant Messaging: The Case of French. PsychNology Journal, 12(1-2), 45 -63 (2014)

Mancera, A., Usos lingüísticos alejados del español normativo como seña de identidad en las redes sociales. https://doi.org/10.1080/14753820.2016.1181435. Bulletin of Spanish Studies 93, 1-25 (2016)

Mas, I. y Zas, L., De lo Necesario a lo Inevitable. Casi Dos Décadas de Código SMS. En: T. Jiménez, B. López, y V. Vázquez (Eds.), Cum corde et in nova grammatica, 585-595. Santiago de Compostela: Universidade (2012)

Monroy, A., Hernández, I.A., y Jiménez, M., Aulas Digitales en la Enseñanza Superior: Caso México. https://doi.org/10.4067/S0718-50062018000500093. Formación Universitaria, (11) 5. 93-104 (2018)

Pascual, J.A., El WhatsApp no Daña la Lengua, Es un Formato Distinto. La Vanguardia. (2013)

Pedrero- Pérez, E.J., Ruíz-Sánchez, J.M., y otros 5 autores, Tecnologías de la Información y la Comunicación (TIC): Uso Problemático de Internet, Videojuegos, Teléfonos Móviles, Mensajería Instantánea y Redes Sociales mediante el MULTICAGE-TIC, Adicciones, 30, 19-32 (2018)

Pinedo, C., El Abuso de WhatsApp y sus Efectos en la Comunicación Juvenil. El País. (2016)

Plester, B., Wood, C., Joshi, P., Exploring the Relationship between Children's Knowledge of Text Message Abbreviations and School Literacy Outcomes, https://doi.org/10.1348/026151008X320507. British Journal of Developmental Psychology, 27, 145-161 (2009)

Prensky, M., H. sapiens digital: From digital immigrants and digital natives to digital wisdom. Innovate, 5 (3) (2009)

Risser, H.S., Virtual Induction: A Novice Teacher's Use of Twitter to Form an Informal Mentoring Network, https://doi.org/10.1016/j.tate.2013.05.001. Teaching and Teacher Education, 35, 25-33 (2013) 
Suárez-Loor, I., El Uso Excesivo del Teléfono Móvil y su Efecto en las Competencias Ortográficas de los Estudiantes del 8vo año de Educación Básica del Colegio Mixto Técnico Santa Elena del Cantón de Santa Elena. Tesis doctoral. Universidad de Guayaquil (2015)

Vázquez-Cano, E., Mengual-Andrés, S., y Roig-Vila, R., Análisis lexicométrico de la especificidad de la escritura digital del adolescente en WhatsApp, https://doi.org/10.4067/S0718-48832015000100005. Revista de Lingüística Teórica y Aplicada 53(1), 83-105 (2015)

Thurlow, C., From statistical panic to moral panic: The metadiscursive construction and popular exaggeration of new media language in the print media, https://doi.org/10.1111/j.1083-6101.2006.00031.x. Journal of Computer Mediated Communication, 11, 667-701 (2006)

Thurlow, C. y Poff, M., The Language of Text-messaging. En: Herring, S. C., Stein, D., Virtanen, T. (Eds.). Handbook of the pragmatics of CMC. Mouton de Gruyter: Boston, MA. 163-190 (2013)

Turner, K. H., Digitalk: A New Literacy for A Digital Generation, https://doi.org/10.1177/003172171009200106, Phi Delta Kappan 92(1), 41-46 (2010) 
